\title{
Capacidad Tecnológica y economía del conocimiento: ubicación del Perú en los rankings internacionales
}

\section{Technological capabilities and the knowledge economy: place of Peru in the international rankings}

\author{
Luis Ponce Vega \\ Universidad Nacional Federico Villarreal, Lima, Perú
}

DOI: https://doi.org/10.33017/RevECIPeru2013.0019/

\section{Resumen}

En las tres décadas pasadas, la humanidad ha sido expuesta a un sinfín de cambios tecnológicos que han transformado la industria, la economía y la sociedad. Para integrar al Perú a la revolución digital en curso, se requiere implementar medidas adecuadas que involucren a la academia, la empresa y el Estado para potenciar nuestra capacidad tecnológica, fomentar la actividad científica organizada, la investigación aplicada y un sistema educativo de punta, pues este es el espacio donde las sociedades industriales y postindustriales generan la mayor parte del conocimiento con potencial productivo.

Sin embargo, suscitar un amplio apoyo a este objetivo nacional requiere, previamente, conocer los aspectos fundamentales y las implicancias del complejo proceso en curso; a saber: ¿Cuál es la naturaleza del cambio científico-técnico y cuáles son sus principales características? ¿Cuáles son los principales modelos de interpretación de dicho proceso? ¿En qué posición se ubica el Perú en el orden de prelación internacional y en qué grupo de países se encuentra? El presente artículo intenta responder estas interrogantes.

Descriptores: Revolución tecnológica global, Sociedad de la Información, Economía del conocimiento, competitividad, innovación, política tecnológica, infraestructura tecnológica y capital humano, indicadores compuestos sintéticos, análisis cluster, barreras e impulsores del cambio tecnológico.

\begin{abstract}
In the past three decades, mankind has been exposed to countless technological changes that have transformed the industry, the economy and society. To join Peru to the ongoing digital revolution, it is required to adopt measures that include the academy, business and government to enhance our technological capacity, applied research and organized scientific activity, as well as a top educational system, because in these areas, industrial and postindustrial societies, together with research and development management, produce most potential productive knowledge.

However, to spark a broad national support to this goal, requires a previous knowledge about the fundamentals (and implications) of the complex ongoing process, namely: What is the nature of technical change and what are its main features? What are the main models of interpretation of this process? In which position is placed Peru in the international rankings, and to what group of countries has joined? This paper attempts to answer these questions.
\end{abstract}

Keywords: Information Society, Knowledge economy, innovation, Global technological revolution, human capital, composite synthetic indicators, barriers and drivers of technological change. 


\section{Metodología}

Para conocer los aspectos fundamentales y las implicancias del complejo proceso en curso que han transformado la industria, la economía y la sociedad, se precisa responder a tres interrogantes clave: ¿cuál es la naturaleza del cambio científico-técnico y cuáles son sus principales características? ¿Estamos frente a cambios científico tecnológico importantes, pero de carácter intermitente, o estamos frente a una revolución industrial, como señala la UNESCO 0 frente a una revolución tecnológica global, como lo indica Anton et al. y lo ratifica Silberglitt et al.?

En segundo lugar, se precisa conocer cuáles son los principales modelos de interpretación de dicho proceso. En tercer lugar, se precisa disponer de indicadores precisos que nos indiquen en qué posición se ubica el Perú en el orden de prelación internacional en materia de capacidad tecnológica y en qué grupo de países se encuentra. Para responder a estas interrogantes se revisará la literatura especializada utilizando los métodos inductivo-deductivo, los cuales, según Mario Bunge [1] son complementarios.

\section{Introducción}

El presente artículo trata de responder las interrogantes arriba señaladas usando los indicadores sintéticos compuestos que miden la capacidad tecnológica de los países y los avances de la economía del conocimiento y que proporcionan rankings internacionales sobre ambos temas. Estos indicadores dan una idea del lugar que ocupa el Perú en el ámbito internacional, a la vez que dan luces sobre los puntos fuertes y débiles del país en materia de desarrollo científico, educativo y tecnológico.

\section{Cambio tecnológico. ¿Intermitente o sostenido?}

En esencia, ¿en qué consisten los cambios técnicos acelerados que han ocurrido en las tres últimas décadas? ¿Son intermitentes, o son sostenidos en el tiempo?

\subsection{Estancamiento Productivo}

A mediados de la década de 1970, se produjo el estancamiento de la producción mundial y la expansión del desempleo en las 15 economías más importantes de la Unión Europea (éste subió de $2 \%$ en 1970 a $11 \%$ en 1994), dando lugar a un estado de ánimo lúgubre, encarnado en la obra de Vivianne Forrester [2] quien reseña la destrucción del trabajo $y$ el fin de un estilo productivo. La prolongada parálisis económica y el creciente desempleo sugerían que las técnicas de producción en masa, asociadas con el fordismo, que utilizaban materias primas tradicionales y energía barata, habían alcanzado sus límites. [3] Es decir, el proceso recesivo en curso respondía a otra lógica y los hechos parecían adecuarse mejor al concepto de destrucción creativa propuesto por Joseph A. Schumpeter en 1943. [4] Por lo tanto, la quiebra de las empresas obsoletas y el desempleo resultante, constituían un paso ineludible para la expansión de los nuevos productos y tecnologías. En este contexto, resultaba lógico que la mitad de las cien empresas más grandes de 1970 ya no existieran el año 2000. [5]

\subsection{Cambio Tecnológico Acelerado. ¿Intermitente o Continuo?}

Los primeros indicios sólidos sobre la naturaleza de las nuevas tecnologías, fueron aportados por Lester Thurow en 1992, quien además identificó a las industrias que ya las estaban empleando: (la microelectrónica, la aviación civil, las máquinas herramientas, la robótica, la biotecnología, las industrias de nuevos materiales, las computadoras, el software y las telecomunicaciones). Identificó, igualmente, su capacidad para disminuir el uso de energía y mano de obra y minimizar la utilización de las materias primas; resaltó, igualmente, sus elevados gastos en Investigación y Desarrollo, así como su capacidad para localizarse en cualquier lugar del mundo, destacando su incidencia en la vida individual y colectiva. [6]

Una década más tarde, el 2001, la abrumadora evidencia acumulada condujo a Anton y asociados a sostener la existencia de una Revolución Tecnológica Global que estaba transformando el mundo contemporáneo; señalando que entre sus principales componentes se incluían a la genómica, la ingeniería biomédica, la clonación, los materiales inteligentes, las manufacturas ágiles, los instrumentos de computación nano-fabricados y los micro-sistemas integrados. [7]

Igualmente, en su Informe Mundial sobre la Ciencia del año 2005, la UNESCO (Organización de la Naciones Unidas para la Educación, la Ciencia y la Cultura) utilizó el concepto de Tercera Revolución Industrial para referirse a los cambios radicales que estaban causando las nuevas tecnologías. "Nuestra época es escenario de transformaciones y cambios 
radicales tan considerables que algunos no vacilan en afirmar que estamos viviendo una tercera revolución industrial -la de las nuevas tecnologías de la información y la comunicación- que va acompañada por un cambio en el régimen de los conocimientos." [8]

Al año siguiente, Silberglitt y asociados confirmaron esta impresión, reseñando los primeros resultados productivos concretos que habían aportado la biotecnología, la nanotecnología, la tecnología de los nuevos materiales y las tecnologías de la información y comunicación. [9] Apenas siete años después, el 2013, James Manyika y sus colegas del Instituto Global McKinsey identificaron más de 100 aplicaciones de las nuevas tecnologías, dejando pocas dudas sobre su potencial creador y destructivo, en el sentido Schumpeteriano.

Como muestra, sus expertos seleccionaron 12 aplicaciones con gran potencial para continuar transformando la sociedad y la producción durante los próximos años: Internet Móvil, automatización del trabajo basado en el conocimiento, internet de las cosas, la tecnología de la nube, los vehículos autónomos o casi autónomos, las impresoras 3D, los materiales avanzados, la exploración y recuperación avanzada de gas y petróleo, la energía renovable, robótica avanzada, la nueva generación de genómica y almacenamiento avanzado de energía. [9]

No hay duda entonces, estamos viviendo una revolución tecnológica global, cuyas manifestaciones parecen no tener fin. [10]

\section{La Sociedad de la Información y la Economía del Conocimiento.}

Una revolución tecnológica global transforma radicalmente las empresas y la forma cómo operan; modificando progresivamente los paradigmas académicos y la organización social. ¿Cómo interpretar estos cambios tecnológicos que vienen de fines del siglo XX?

\subsection{La Sociedad de la Información y las tecnologías de la información}

Dada la visibilidad que han adquirido las tecnologías de la información, el concepto más difundido de los cambios tecnológicos, fue el de la Sociedad de la Información. Entre los aportes más difundidos resalta la Tercera Ola de Alvin Toffler, (1980), que afirma que la revolución de la información había dado lugar a la tercera ola (de cambios tecnológicos) que estaba transformando la sociedad y la economía mundial. Igualmente, el Advenimiento de la Sociedad Post-Industrial, de Daniel Bell, 1973, señaló las características de esta Sociedad cuando las tecnologías de la información, aplicadas al transporte y la economía, alcanzaban una gran difusión. Así, el concepto de sociedad post-industrial fue visto como un sinónimo de la sociedad de la información. [11]

Para la década de los 90 , ya se pensaba que la Sociedad de la Información tendría tanta importancia como la sociedad industrial. Citando a John Naisbitt, autor de las mega-tendencias, se afirmó que la tecnología de las computadoras tendría el mismo impacto para la Edad de la Información, que la mecanización en la Revolución Industrial. [12] El prestigio de la Sociedad de la Información se consolida con la influyente obra de Castells quien (desde Berkeley) difunde sus tesis sobre el rol de los medios de comunicación y el enorme efecto creadordestructor (en el sentido schumpeteriano) de la revolución tecnológica del Silicon Valley. Su extraordinario dominio del tema culmina con su obra maestra, la Era de la Información.

\subsection{La Sociedad del Conocimiento}

Conforme las fuentes de innovación de la sociedad empiezan a derivarse más de la investigación y el desarrollo (I+D) y conforme una mayor proporción del PBI y del empleo empiezan a residir en el conocimiento, se introdujo el concepto de sociedad del conocimiento, usado por autores asociados con la UNESCO. [13] Si en el nuevo marco teórico, el principal recurso de la sociedad era su personal científico, las políticas públicas debían tener en cuenta la distribución de dicho personal por sectores (industria, gobierno, universidad) y por funciones (producción, investigación, enseñanza).

\subsection{La Economía del Conocimiento y sus Cuatro Pilares.}

Sin embargo, para fines prácticos, la Economía Basada en el Conocimiento conjuga cuatro elementos clave; un régimen de incentivos económicos, el acceso a la educación, un sistema efectivo de innovación y una infraestructura informática moderna. Además, la Economía del Conocimiento goza de una ventaja adicional, dispone de un índice que da cuenta de los avances logrados por cada país en las cuatro áreas arriba descritas. [12] 


\section{Indicadores de Innovación y Desarrollo Tecnológico.}

Debido a la complejidad que ha adquirido la tecnología, la gente posee más conciencia de que los indicadores tradicionales, como el número de patentes registradas 0 las exportaciones de productos de alta tecnología, examinados aisladamente, no miden adecuadamente las competencias tecnológicas de los países. Por tal razón, se propuso la evaluación conjunta de los indicadores, porque una batería de indicadores ofrece una imagen más completa de la que podría ofrecer un solo indicador examinado aisladamente. [14]

\section{Gracias a los indicadres compuestos sintéticos} se puede medir la importancia que ha adquirido la tecnología en nuestra estructura económica; dan una rápida idea de los componentes que conforman las capacidades tecnológicas de nuestro país y muestran el lugar que ocupa en los rankings internacionales en materia de capacidad tecnológica.

Las organizaciones internacionales, como las Naciones Unidas (UNDP, UNIDO, UNCTAD), la Organización para la Cooperación y el Desarrollo Económico (OCDE), el Banco Mundial y el Foro Económico Mundial, elaboraron indicadores compuestos sintéticos. Aunque también se debe relevar al indicador ArCo, elaborado por dos investigadores italianos, que logró una amplia difusión debido a su simplicidad, cobertura y efectividad. [15]

\subsection{El Índice Compuesto de Capacidades Tecnológicas, ArCo. 1990 y 2000}

El indicador ArCo fue elaborado por Daniele Archibugi y Alberto Coco para 162 países, con la finalidad de identificar la brecha tecnológica entre los países, el incremento del capital humano y el aumento de la infraestructura científica entre 1990 y 2000.

Este indicador incluye las tres dimensiones más importantes para construir las capacidades tecnológicas de un país: El desarrollo de la tecnología, la infraestructura tecnológica y el avance de las capacidades humanas.

A su vez, estos componentes están conformados por ocho sub-indicadores. El primer componente incluye las patentes y los artículos científicos en revistas indexadas. El segundo, comprende la penetración de Internet y de la telefonía, así como el consumo de electricidad. El tercero, incluye la matrícula en estudios de ciencia e ingeniería (sector terciario); los años promedio de escolaridad y la tasa de alfabetización.

\section{División del mundo en cuatro grupos de países}

El índice de tecnología ArCo clasificó los 162 países en cuatro grupos. Los primeros 25 fueron catalogados como los países líderes, porque eran capaces de crear y sostener la innovación tecnológica y porque concentraban la mayor parte de la creación mundial de tecnología.

Los países ubicados entre el puesto 26 y 50 fueron designados como líderes potenciales, porque invertían en la formación de capacidades humanas y en el desarrollo de la infraestructura tecnológica estándar de sus países, pero que aún no habían logrado muchos resultados tangibles en materia de innovación.

Los países ubicados entre el puesto 51 y 111 fueron clasificados como países recién arribados. Es el grupo más numeroso, está compuesto por los países que, de una u otra manera, estimulan el crecimiento de la infraestructura tecnológica y la formación de las capacidades humanas de sus respectivos países, en paralelo a sus actividades tradicionales de desarrollo.

En este grupo se encuentra el Perú y nueve países latinoamericanos que han ganado posiciones en la década bajo estudio, (1990-2000), como Panamá, Venezuela, Costa Rica, México, Jamaica, Colombia, Brasil, Paraguay y Bolivia. Estos países construyeron su infraestructura tecnológica (a tasas de crecimiento de alrededor de $20 \%$ ), pero no lograron que las capacidades humanas de su población crecieran de manera tan eficaz (no superaron el 10\%).

Según ArCo, el Perú ocupa el puesto 67 sobre el total de 162 países que conforman su base de datos; lo cual nos ubica en el $40 \%$ superior.

Finalmente, los cincuenta y un países que van del puesto 112 al 162 forman parte del grupo de los marginados. No tienen acceso a las tecnologías viejas, como la electricidad y la telefonía. Aquí están las economías de bajos ingresos de África y del sudeste asiático. 


\subsection{El Índice de la Economía del Conocimiento (KEI)}

El Índice de la Economía del conocimiento (KEI) es elaborado por el Banco Mundial para 146 países en base a 81 variables. Este indicador agrupa a los países en tres categorías.

En la primera están los países que se encuentran en las etapas avanzadas de desarrollo; aquí se encuentra la mayoría de las economías de la OCDE y las de reciente industrialización de Asia Oriental. En el Gráfico $\mathrm{N}^{\circ} 1$, estos países se hallan a la derecha. Igualmente, los que están por encima de la diagonal son los que han logrado progresos entre el año base (1995) y el 2012. En cambio, los que están por debajo de la diagonal han sufrido un retroceso entre los dos años mencionados.

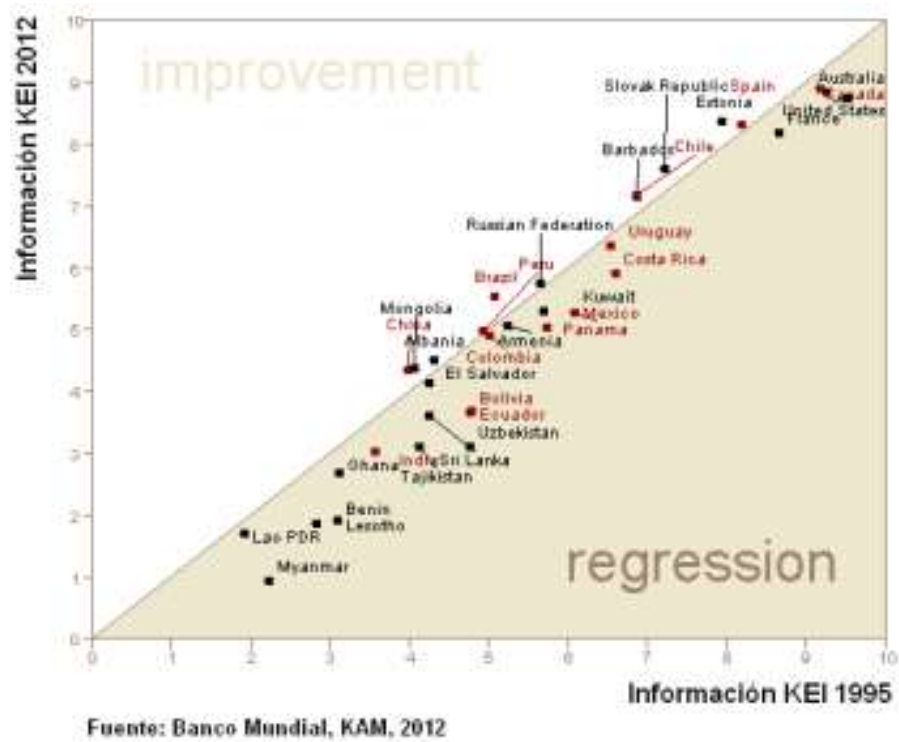

Figura 1: Índice de la Economía del Conocimiento (1995-2012)

En el segundo, están los países en tránsito a la economía del conocimiento, que incluye a los países de ingresos medios de Europa y Asia Central, Asia del Este, Oriente Medio, el Norte de África y América Latina. [16]

El Perú se halla en este grupo, ubicado en el puesto 74 (sobre 146 países que comprende la base de datos). Como se puede observar en el Gráfico 1, el Perú se encuentra por encima de la diagonal, lo cual indica que ha registrado progresos entre el año inicial y el 2012.

El tercer grupo incluye a los países que recién han iniciado el camino para convertirse en una economía del conocimiento. Aquí están los países de bajos ingresos de África y del sudeste asiático.

\section{Ubicación del Perú en la Economía del Conocimiento}

Según el índice de la Economía del Conocimiento, entre 1995 y el 2012 el Perú muestra un ligero avance en términos agregados. De acuerdo con la Tabla 1, su puntaje se incrementó de 4.93 (en 1995) a 5.01 el 2012, (este índice tiene un valor máximo de 10 puntos). En cuanto a su posición en el ranking internacional, el país mejoró su posición al ascender del puesto 80 (1995), al puesto 74 (2012), sobre un total de 146 países. Este resultado nos ubica justo en la mitad de la muestra.

Tabla 1: Perú: Índice de la Economía del Conocimiento. (1995 - 2012)

\begin{tabular}{|c|l|c|}
\hline \multirow{2}{*}{1995} & IEC & 4,93 \\
\cline { 2 - 3 } & Ranking & 80 \\
\hline \multirow{2}{*}{2000} & IEC & 4,99 \\
\cline { 2 - 3 } & Ranking & 66 \\
\hline \multirow{2}{*}{2008} & IEC & 4,64 \\
\cline { 2 - 3 } & Ranking & 74 \\
\hline \multirow{2}{*}{2012} & IEC & 5,01 \\
\cline { 2 - 3 } & Ranking & 74 \\
\hline
\end{tabular}

Fuente: Banco Mundial

En cambio, los resultados parciales obtenidos en el sub-índice "Tecnologías de la Información y la Comunicación" constituyen una señal de preocupación, pues el 2012 se obtuvieron apenas 5.18 puntos. Este resultado nos está alertando de un ligero retroceso con respecto a 1995, cuando se obtuvieron 5.59 puntos. Sin embargo, los resultados que deben causar más preocupación son los referentes al sub-índice "Innovación", puesto que no sólo constituye el más bajo de los resultados parciales obtenidos el 2012 (4.11 puntos) sino que, además, significó un retroceso con respecto al modesto 4.36 obtenido en 1995.

Al considerar todos los resultados parciales, se puede observar que el Perú tuvo su mejor performance en el rubro incentivos económicos y régimen institucional (con 7.17 puntos el 2012, versus 3.08 en 1995) y en el rubro de educación (con 5.68 el 2012). Aunque se debe notar que esta última cifra representa una disminución con respecto a 1995. 


\section{Tecnología y Producción. Panorama al 2020}

Finalmente, que se puede decir con respecto al futuro. ¿Qué destino le depara al Perú los estudios de prospectiva, en especial al 2020 , una fecha muy cercana al bicentenario de la República?

Un ejercicio de prospectiva para el 2020 realizado por Silberglitt et al., (2006) plantea un escenario en el cual las tendencias tecnológicas actuales se mantendrán. Es decir, las nuevas tecnologías continuaran modificando el actual escenario productivo internacional, integrando múltiples disciplinas científicas, con efectos profundos en la sociedad. Pronostica también que el mundo estará conformado por cuatro mundos, de una manera muy parecida a la clasificación planteada por el indicador ArCo, despertando la curiosidad por saber en cuál de estas agrupaciones podría encontrarse el Perú el 2020. [17]

\section{Resultados y discusión}

Existe una revolución tecnológica-productiva en curso que está transformando la sociedad, la industria y la economía que, además, ha convertido en obsoletos los modelos de interpretación societal y de desarrollo económico vigentes a partir de la postguerra de la segunda guerra mundial.

El examen de las teorías del cambio tecnológico y la evolución de los indicadores compuestos sintéticos nos han permitido examinar la importancia de los modelos societales-culturales favorables al cambio tecnológico y a la economía del conocimiento. En la actualidad se propone a la Sociedad de la Información y la Economía del Conocimiento como intérpretes de los cambios en curso.

Debido a la complejidad que ha adquirido la tecnología, se utilizan los indicadores compuestos sintéticos porque brindan una mejor visión del avance de cada país en esta materia, así como de las diferencias que existen entre ellos. De igual manera, la evaluación conjunta de varios indicadores simultáneamente permite combinar y ponderar los elementos de juicio que aporta cada uno de ellos, porque una batería de indicadores ofrece una imagen más completa de las diferencias que existen entre los países, que la que podría ofrecer un solo indicador, examinado aisladamente.

El examen de los principales indicadores del cambio tecnológico nos permite configurar la posición que juega actualmente el Perú en el panorama internacional, pues todos los indicadores lo ubican en la mitad del total de países examinados o en el $40 \%$ superior.

Así tenemos que el indicador ArCo ubica al Perú en el lugar 67 sobre una base total de 162 países, ubicándolo en el tercer grupo de países (los recién llegados). Igualmente, el Índice de la Economía del Conocimiento ubica al Perú en el puesto 74 de un total de 146 países que conforman la base de datos del Banco Mundial, (en estos años, la economía nacional creció en $18.21 \%$ ) La misma ubicación se mantiene en el caso del ejercicio de prospectiva al 2020.

El examen realizado permite precisar las fortalezas y debilidades del camino emprendido por nuestro país. Proporciona, igualmente, las bases para un diseño realista de las estrategias que permitan que los directivos de la academia, las empresas y el Estado puedan establecer las bases del modelo de la triple hélice y las reformas que configuren una labor más estrecha entre los centros de investigación (universitarios, empresariales y del Estado).

\section{Conclusiones}

En base a los arriba expuesto, queda justificado el establecimiento de los vicerrectorados de investigación, el desarrollo de los parques científicotecnológicos, la promoción de las gerencias de Investigación y Desarrollo, brazos promotores de la I+D en las empresas, y la constitución de un ente similar al USPTO de Estados Unidos o a la Oficina Española de Patentes y Marcas, ligado al MINCETUR, que permita implementar proyectos de exportación, apoyados por proyectos de investigación entre la academia y la empresa innovadora, a fin de aprovechar los TLC y los acuerdos internacionales de marcas y patentes suscritos por el Perú.

\section{Agradecimientos}

Se agradece el aporte de los expertos del CONCYTEC y de la Comisión de Ciencia, Innovación y Tecnología del Congreso de la República; igualmente, las sugerencias de los especialistas que asistieron al Encuentro Científico Internacional de verano 2014.

\section{Referencias}

[1] Mario Bunge. La ciencia, su método y su filosofía. Santa Fe de Bogota. Panamericana, 1996. 
[2] Vivianne Forrester. L'Horreur Économique. Editions Fayard, 1996

[3] Luis Ponce Vega. Perú Republicano: Cambio Tecnológico y Tendencias de Largo Plazo. En: CEPLAN. La Economía en el Perú Republicano. Documentos de Trabajo $\mathrm{N}^{\circ} 3$. Lima, CEPLAN, 2010. (pp. 42-80).

[4] Joseph A. Schumpeter. Capitalism, Socialism, and Democracy. London, Taylor \& Francis, 2003. (p.83). Se publicó por primera vez en 1942.

[5] Richard N. Foster \& Sarah Kaplan. Creative destruction - why companies that are built to last underperform the markets - and how to transform. New York, Currency, 2001.

[6] Lester Thurow. Head to Head. The coming economic battle among America, Japan and Europe. New York: Harper Collins. 1992.

[7] Philip S. Anton, Richard Silberglitt, James Schneider. The global technology revolution: bio/nano/materials trends and their synergies with information technology by 2015. Santa Mónica: Rand Corporation, 2001.
[8] UNESCO. Science Report 2005. Paris: UNESCO, 2005.

[9] R. Silberglitt, P.S. Anton and D.R. Howell. The Global Technology Revolution 2020. Bio, Nano, Materials, Information trends, drivers, barriers and social implications. Santa Mónica: Rand Corporation, 2006.

[10] James Manyika, Michael Chui, Jacques Bughin, Richard Dobbs, Peter Bisson y Alex Marrs. Disruptive technologies: Advances that will transform life, business, and the global economy. McKinsey Global Institute. 2013

[11] F. Webster. Theories of Information Society. $3^{\text {rd }}$. edition. London: Routledge ,2006. (p.11)

[12] John Naisbitt. Mind Set. New York, New York, USA, Harper Collins, 2006

[13] Daniele Archibugi y Alberto Coco. A New Indicator of Technological Capabilities for Developed and Developing Countries (ArCo). World Development Vol. 32, No. 4, pp. 629654, 2004

[14] Chen, Derek H.C. y Carl J. Dahlman. (2005). The Knowledge Economy, the KAM Methodology and World Bank Operation. World Bank Institute Working Paper No. 37256 October 19, 2005.

E-mail: ponceve@gmail.com 